\title{
The inventor of modern science
}

\section{James Bradley laid the foundations of modern science in his aunt's attic. His impressively precise astronomical measurements gave birth to experimental physics as we know it. The first in our series of millennium essays.}

\section{Freeman J. Dyson}

M odern science began in 1729 , when it became based on measurements of high precision. This makes James Bradley, who did the first high-precision measurements, the inventor of modern science. He was the first to understand that accurate measurement requires meticulous monitoring and control of possible sources of error. He was the first to record temperature and barometric pressure whenever he made an observation.

In 1729, Newton had been dead for two years. Only 120 years had passed since Galileo's little telescope had first been pointed at Jupiter and its satellites. Bradley had the good luck to have a widowed aunt who owned the house where she lived in Wanstead, east London. The house had a high roof, and the aunt allowed him to cut a hole in her roof and use the upper part of the house as an observatory. Her husband had also been a devotee of astronomy, so she was used to this sort of madness. Under the hole, Bradley suspended a slender three-inch refracting telescope, with a brass circular arc along which the eyepiece of the telescope could be moved with a micrometer screw.

The eyepiece was focused simultaneously

Aberration becomes the norm: Bradley's careful measurements proved that the Earth orbits the Sun, and established the now traditional working methods of science.

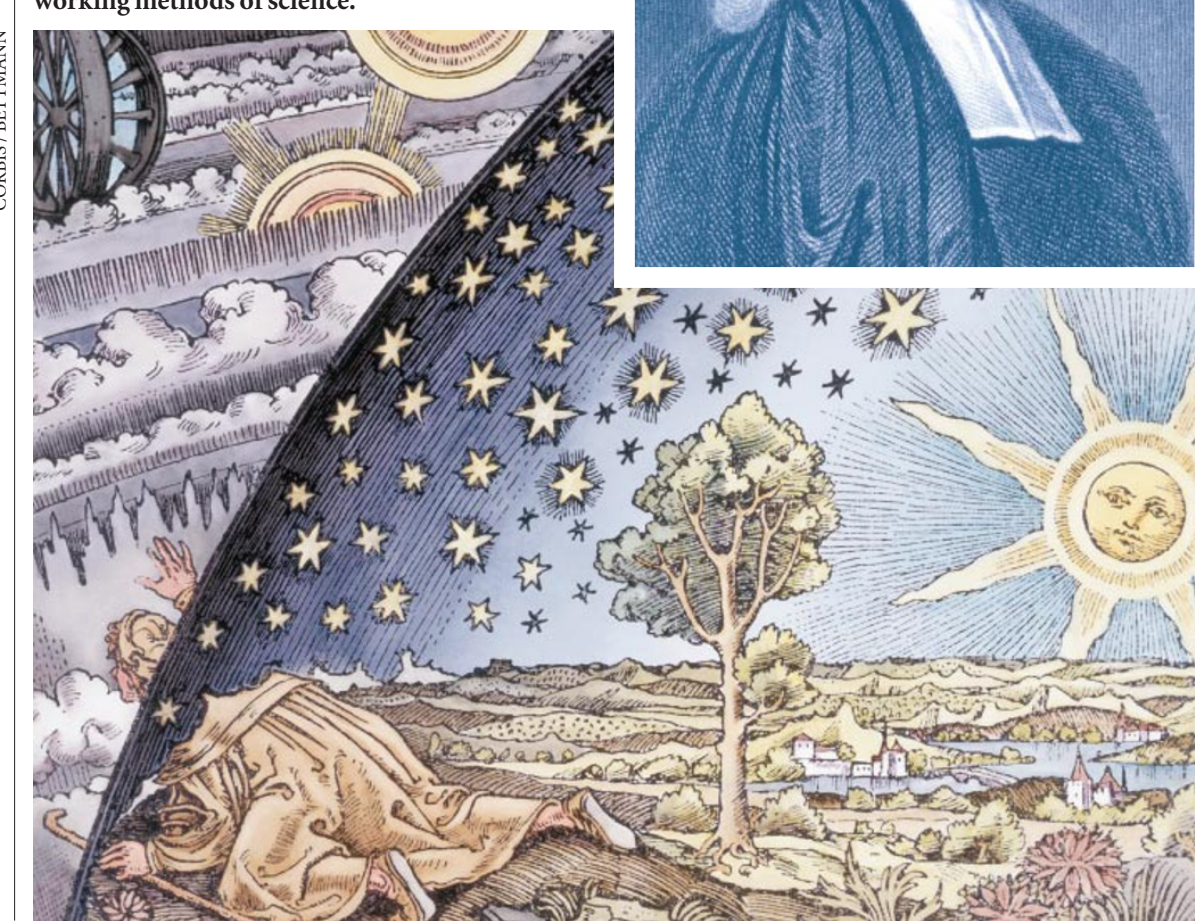

on a star overhead and on a fine crosswire. Bradley could move the star exactly onto the crosswire with the micrometer screw, and then read off the angle on the brass arc. At one place on the arc there was a notch leaving space for a vertical plumb-line made of wire with one hundredth of an inch diameter. We must assume that he had his aunt well trained so that she did not slam doors while observations were in progress.

Using his plumb-line and his micrometer screw, Bradley was able to measure the angle between a star and the local vertical with sixfigure accuracy, with an error of one or two parts per million. He was the first person to measure anything with six-figure accuracy. He measured angles a hundred times more accurately than the astronomers of Newton's time. That was how he discovered aberration.

Aberration is the displacement of the image of a star in the sky due to the speed of the Earth in its orbit around the Sun. Bradley found that all the stars moved in elliptical paths in the sky, coming back to their original

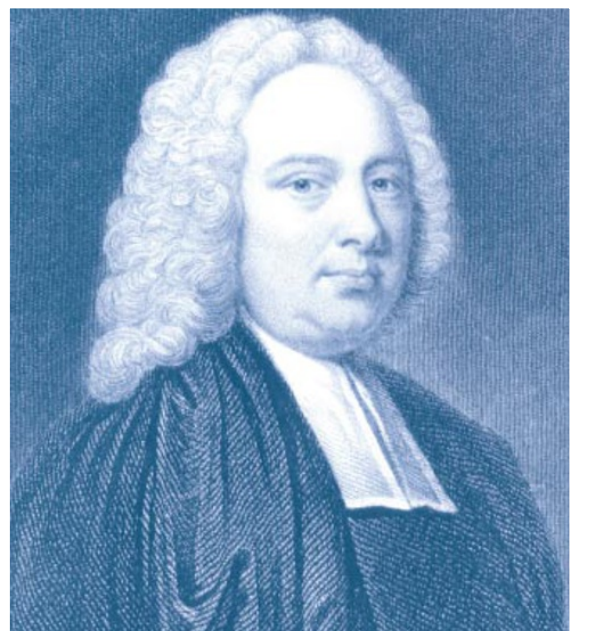
precisely observed celestial motions. One hundred and fifty years after Bradley, Albert Michelson and Edward Morley attempted to measure the second-order aberration of light caused by the Earth's motion through the ether, and by their negative result led the way to the theory of relativity.

Finally, after 200 years, the tradition of six-figure accuracy begun by Bradley led to the modern flowering of experimental physics, with Isidor Rabi's molecular beam apparatus probing the resonances of atoms as precisely as Bradley's micrometer probed the motions of the stars.

Freeman J. Dyson is at the Institute for Advanced Study, Princeton, New Jersey 08540, USA. 